\title{
Structure of the DBL3x domain of pregnancy-associated malaria protein VAR2CSA complexed with chondroitin sulfate A
}

\author{
Kavita Singh ${ }^{1}$, Apostolos G Gittis ${ }^{1}$, Phuc Nguyen ${ }^{1}$, D Channe Gowda ${ }^{2}$, Louis H Miller ${ }^{3}$ \& David N Garboczi ${ }^{1}$
}

\begin{abstract}
Plasmodium falciparum-infected erythrocytes bind to chondroitin sulfate A (CSA) in the placenta via the VAR2CSA protein, a member of the $\boldsymbol{P}$. falciparum erythrocyte membrane protein-1 family, leading to life-threatening malaria in pregnant women with severe effects on their fetuses and newborns. Here we describe the structure of the CSA binding DBL3x domain, a Duffy bindinglike (DBL) domain of VAR2CSA. By forming a complex of DBL3x with CSA oligosaccharides and determining its structure, we have identified the CSA binding site to be a cluster of conserved positively charged residues on subdomain 2 and subdomain 3. Mutation or chemical modification of lysine residues at the site markedly diminished CSA binding to DBL3x. The location of the CSA binding site is an important step forward in the molecular understanding of pregnancy-associated malaria and offers a new target for vaccine development.
\end{abstract}

Plasmodium falciparum infection during pregnancy results in the sequestration of infected erythrocytes in the placenta causing maternal anemia as well as low birth weight, premature birth and increased infant mortality (for reviews, see refs. 1-4). Irrespective of gender, adults living in endemic areas generally acquire a degree of immunity that prevents severe malaria, but pregnant women, despite having pre-existing protective immunity, are susceptible to severe disease, especially during their first pregnancy. Consequently, pregnancyassociated malaria poses a risk to millions of women across the globe every year.

Pathogenesis of malaria in pregnant women is primarily due to binding of infected erythrocytes to CSA in the placenta ${ }^{5-7}$. The parasite modifies the surface of infected erythrocytes to express P. falciparum erythrocyte membrane protein-1 (PfEMP1). PfEMP1 molecules are encoded by 50-60 parasite var genes and are involved in infected erythrocyte binding (sequestration) in the venules of many organs including the placenta. One var gene, var2csa, encodes the VAR2CSA PfEMP1 that is primarily responsible for binding to $\mathrm{CSA}^{8}$. VAR2CSA is upregulated in infected erythrocytes that are either isolated from the placenta ${ }^{9}$ or selected in vitro to bind to $\mathrm{CSA}^{8}$. Furthermore, the ability of infected erythrocytes to adhere to CSA is lost ${ }^{10}$ or reduced ${ }^{11}$ when the var2csa gene is disrupted. Of the six DBL domains of VAR2CSA, at least three, DBL2x, DBL3x and DBL6e, bind $\mathrm{CSA}^{12,13}$. In the laboratory, the binding of infected erythrocytes to placental chondroitin sulfate proteoglycan can be maximally inhibited by dodecasaccharides prepared from bovine tracheal CSA ${ }^{14}$.
In geographically diverse malaria endemic areas, antibodies that are naturally acquired by women during previous pregnancies block the binding of infected erythrocytes to $\mathrm{CSA}^{15}$. These findings suggest that epitopes expressed by various placental isolates are conserved and that a vaccine against pregnancy-associated malaria is possible. Owing to its sequence conservation, the DBL3x domain of VAR2CSA is considered to be a major target for vaccine development ${ }^{1}$. With this in mind, we have determined the structure of DBL3x, one of the CSA binding domains of $P$. falciparum VAR2CSA, and explored the structural basis of its binding to CSA by soaking and cocrystallization with CSA oligosaccharides of various sizes. In addition, we have investigated the binding of CSA to DBL3x through the use of chemical modification, mutation, flow cytometry and isothermal titration calorimetry (ITC). Considered together, the data from these experiments reveal the location of the CSA binding site and the nature of its interaction with DBL3x.

\section{RESULTS}

\section{Overall structure of DBL3x}

We overexpressed the DBL3x domain (residues 1220-1580, GenBank AAQ73926) of the VAR2CSA protein from the A4 strain ${ }^{12}$ of $P$. falciparum in Escherichia coli as insoluble inclusion bodies (Methods). DBL3x was refolded in vitro to its functional form, was then purified and migrated as a monomer during size-exclusion chromatography. We determined the DBL3x crystal structure, both

${ }^{1}$ Structural Biology Section, Laboratory of Immunogenetics, National Institute of Allergy and Infectious Diseases, National Institutes of Health, 12441 Parklawn Drive, Rockville, Maryland 20852, USA. ²Department of Biochemistry and Molecular Biology, Pennsylvania State University College of Medicine, 500 University Drive, H171, Hershey, Pennsylvania 17033, USA. ${ }^{3}$ Malaria Vaccine Development Branch, National Institute of Allergy and Infectious Diseases, National Institutes of Health, 5640 Fishers Lane, Rockville, Maryland 20852, USA. Correspondence should be addressed to K.S. (ksingh@niaid.nih.gov) or D.N.G. (dgarboczi@niaid.nih.gov). 

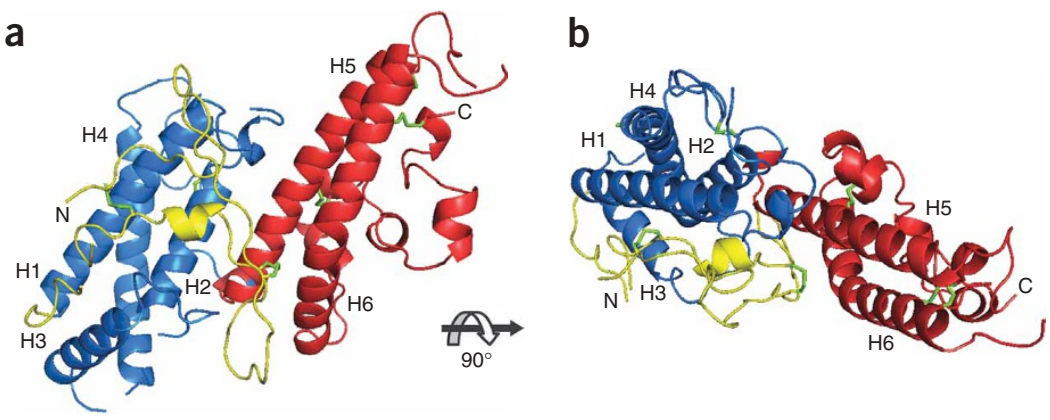

Figure 1 Views of the overall structure of the DBL3x domain. (a) DBL3x is composed of subdomain 1 (yellow), subdomain 2 (blue) and subdomain 3 (red). Subdomain 2 has four helices $(\mathrm{H} 1-\mathrm{H} 4)$ and subdomain 3 has two long helices (H5 and H6). Disulfide bonds (green) link cysteine residues within each subdomain. (b) After a reorientation of $90^{\circ}$, this view of the 'top' of the molecule reveals the thin dimension of the DBL3x domain.

alone and bound to CSA oligosaccharides from four to twelve monosaccharides in length.

The DBL3x structure has three subdomains (using the nomenclature of ref. 16; Fig. 1). The first subdomain (residues 1220-1292; Fig. 1, yellow) lacks regular secondary structure except for a single turn of helix and is held together by two disulphide bonds between Cys1230-Cys1273 and Cys1251-Cys1264. Subdomain 2 (residues 1293-1444) contains four helices (H1-H4) connected by four loops (Fig. 1, blue). An unpaired cysteine (Cys1418) in helix H4 reacted with cystamine during refolding, gaining a cysteamine adduct that we observed in the electron density map and confirmed by MS. The C-terminal portion (residues 1424-1444) of subdomain 2 forms an extended structure that connects to the third subdomain. Cys1437 forms a disulfide bond with Cys1344 on helix H2.

Subdomain 3 (residues 1445-1580) (Fig. 1, red) has two long antiparallel helices, H5 (residues 1449-1476) and H6 (residues 1499-1529), that are connected to each other by a large loop (residues 1477-1498) and that make contacts with subdomains 1 and 2 . The C-terminal portion of subdomain 3 (residues 1563-1580) forms a flat structure of small helices connected by short linker regions. Subdomain 3 contains four disulfide bonds: Cys1462-Cys1546, Cys1476-Cys1501, Cys1505-Cys1574 and Cys1486-Cys1576 (Supplementary Table 1 online). Near the $\mathrm{C}$ terminus, the bond between Cys1486 and Cys1576 was not visible and, presumably, was disordered in the crystal. In addition, nine N-terminal residues, three C-terminal residues and loop residues 1279-1285, 1327-1337, 1387-1397 and 1486-1494 were disordered and not visible in the electron density.

\section{Comparison with known DBL structures}

The prediction that the DBL domains would have a conserved protein fold was made when the var genes were first cloned, on the basis of the observation of conserved cysteines and aromatic residues (Trp, Tyr, Phe $)^{17-19}$. The structures of three other DBL domains have been determined from two proteins that are not PfEMP1 family members: the $P$. falciparum erythrocyte binding antigen (EBA)-175, which has two DBL domains F1 and F2 (PDB 1ZRL) ${ }^{20}$, and the Plasmodium knowlesi $(\mathrm{Pk}) \alpha$-DBL protein, which has one $\mathrm{DBL}$ domain (PDB $2 \mathrm{C} 6 \mathrm{~J})^{16}$. The structure of the PfEMP1 DBL domain reported here, DBL3x, adds to the evidence that PfEMP1 domains have the conserved DBL protein fold. Structure-based sequence alignments of DBL3x with EBA-175 and Pk $\alpha$-DBL show conserved cysteines amid a few other conserved residues (Supplementary Fig. 1 online). We also observed conserved helices in the absence of substantial sequence identity. Structural superimpositions of DBL3x and the three other DBL domains yielded r.m.s. deviations on all $\mathrm{C} \alpha$ atoms for $\mathrm{F} 1, \mathrm{~F} 2$ and $\mathrm{Pk} \alpha-\mathrm{DBL}$ of $2.8 \AA$, $2.7 \AA$ and $2.2 \AA$, respectively. Overlays of the four structures based on the structural superimposition of the long helices in subdomain 3 made it clear that each DBL domain has its three subdomains positioned differently (Supplementary Fig. 2 online). Differences in subdomain positions relative to each other probably contributed to our finding that only the Pk $\alpha$-DBL structure (r.m.s. deviation $2.2 \AA$ ) yielded convincing solutions when used as a model in several molecular replacement programs. Calculated phases from the best molecular replacement solutions, however, were poor, and it was necessary to improve them through multicrystal averaging (Methods).

\section{Location of the CSA binding site}

To obtain crystals of the complex between DBL3x and CSA, DBL3x was cocrystallized or soaked with size-fractionated 6-O-desulfated oligosaccharides prepared from enzymatic digestion of bovine tracheal CSA. We measured X-ray data from 50 crystals, 35 of which were soaked or grown with carbohydrates, resulting in two space groups $\left(P 22_{1} 2_{1} 2_{1}\right.$ and $\left.P 2_{1}\right)$, each showing two sizes of unit cell (Methods). The refined structure of the native protein was used as a model to obtain molecular replacement solutions for each of the DBL3x crystals complexed with CSA that yielded X-ray data sets. We subjected these solutions to crystallographic refinement and examined the resulting electron density maps. In ten data sets from crystals that included tetra-, hexa-, octa- or dodecasaccharides, we observed

a

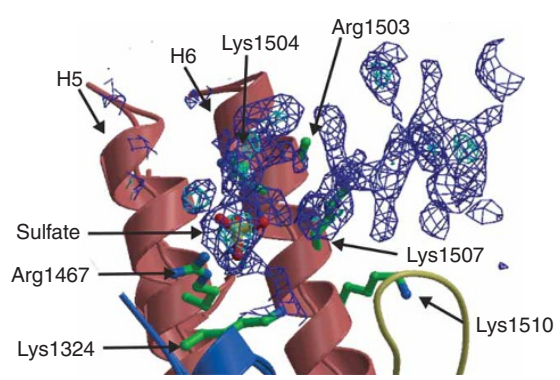

b
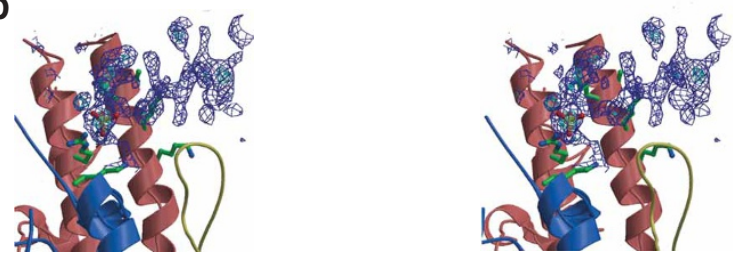

Figure 2 The CSA electron density. (a) View of the CSA electron density near helices $\mathrm{H} 5$ and $\mathrm{H} 6$. Residues with side chains within $5 \AA$ of the density are identified. A sulfate group (sulfur, yellow; oxygen, red) has been placed in the density and is coordinated by the side chains of Lys1324, Arg1467 and Lys 1504 . The $2 F_{0}-F_{\mathrm{c}}$ map (dark blue) is contoured at $0.9 \sigma$ and the $F_{0}-F_{c}$ map (cyan) is contoured at $2.5 \sigma$. For clarity, Lys 1327 is not shown. (b) Stereo view showing the CSA density, a sulfate group and the side chains of nearby residues. The DBL3x model without ligands was used to calculate phases for these maps. 
Figure 3 The lysine residues of DBL3x are important for binding to CSA. (a) DBL3x binds to $\mathrm{CHO}$ cells that express CSA on their surface in a flow cytometry assay ('DBL3x'). The preincubation of $D B L 3 x$ with bovine tracheal CSA ('DBL3x + CSA') or with a CSA hexasaccharide derived from bovine tracheal CSA ('DBL3X + Hexa') reduces DBL3x binding to the cells to the control level observed with the anti-DBL3x antibody alone (gray shaded peak). Preincubation with CSC had no effect on DBL3x binding to the cells (not shown). (b) DBL3x dimethylated on each of its lysine residues ('Methylated DBL3x') does not bind to the CSA-expressing cells, yielding a peak similar to that of the anti-DBL3x antibody alone (gray shaded peak). Immunoblotting control experiments showed that DBL3x methylation did not block the binding of the anti-DBL3x antibody. (c) Both the DBL3x mutant with Lys1507 replaced by alanine (purple peak) and the DBL3x mutant with both Lys1507 and Lys1510 replaced by alanine (green peak) show reduced binding to $\mathrm{CHO}$ cells expressing CSA compared with wild-type DBL3x (magenta peak).
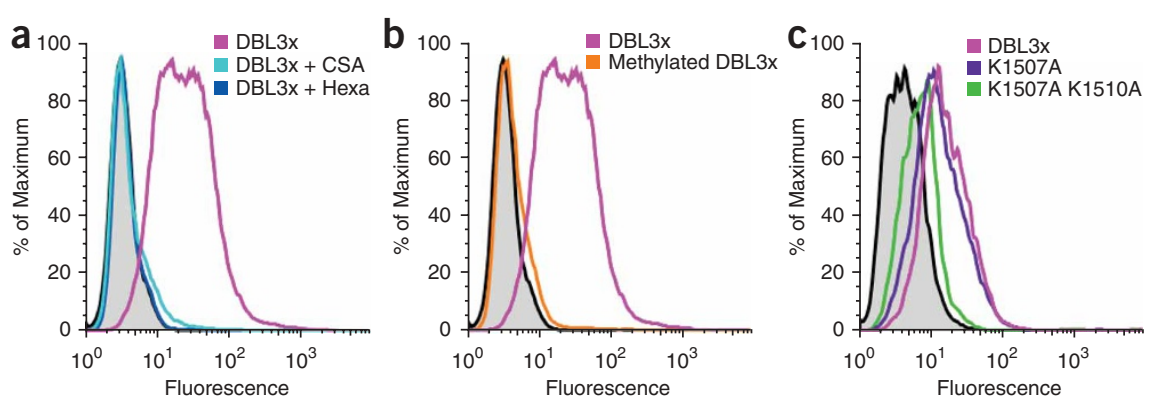

electron density that could not be explained by protein atoms. The extra electron density was seen in $2 F_{\mathrm{o}}-F_{\mathrm{c}}$ maps contoured at $1.0 \mathrm{\sigma}$ and in $F_{\mathrm{o}}-F_{\mathrm{c}}$ maps contoured at $2.5 \sigma$ above the mean density. The density appeared in the same region of DBL3x, whether cocrystallized or soaked, with various sizes and concentrations of oligosaccharide (Fig. 2). We observed no extra density in crystals without added oligosaccharide, excluding the possibility that the density was due to HEPES, polyethylene glycol or water molecules. The extra electron density was of a size that could fit four to six monosaccharides. Residues that are within $5 \AA$ of the CSA density are Lys1324 and Lys1327 of subdomain 2, Arg1467 of helix H5 of subdomain 3, and Arg1503, Lys1504, Lys1507 and Lys1510 of helix H6 of subdomain 3. One strong region of density $\left(1.5 \sigma\right.$ in $2 F_{\mathrm{o}}-F_{\mathrm{c}}, 3.0 \sigma$ in $\left.F_{\mathrm{o}}-F_{\mathrm{c}}\right)$, which we observed in every data set with carbohydrate density, is chemically consistent with a sulfate ion that seems to be coordinated by Lys1324, Arg1467 and Lys1504 (Fig. 2).

These areas of extra electron density appeared only when DBL3x crystals were soaked with CSA oligosaccharides or when crystals were grown in the presence of CSA oligosaccharides. There was a tendency for crystals with carbohydrate density to show the larger unit cell dimensions, whereas crystals without extra density showed the smaller unit cell dimensions. The binding of oligosaccharide seems to change the packing of DBL3x molecules in the crystal, which is consistent with the reduced resolution limit and overall diffraction quality of the complex crystals compared to native crystals.

\section{The lysines of DBL3x are required for binding CSA}

The binding of the DBL3x domain to CSA was assessed using flow cytometry, which showed that the recombinant DBL3x domain recognized CSA expressed on the surface of CHO-K1 cells (Fig. 3, peaks in magenta). The binding of DBL3x to the cells was inhibited by preincubating DBL3x with soluble bovine tracheal CSA, the starting material for the preparation of the oligosaccharides used in the crystals (Fig. 3a, cyan peak). CSA hexasaccharides prepared as described in Methods also inhibited the binding of DBL3x to the CSA expressed by the cells (Fig. 3a, blue peak). Preincubation of DBL3x with chondroitin sulfate $\mathrm{C}$ (CSC) did not inhibit DBL3x binding to the cells (data not shown).

The involvement of lysines in the binding of DBL3x to CSA was confirmed by flow cytometry (Fig. 3b). A version of the DBL3x protein in which all of the lysines were chemically modified by reductive methylation did not bind to CSA on the CHO-K1 cells (Fig. 3b, orange peak). The methylation of lysines is a mild chemical modification that does not change the charge on the lysine side chain, but adds two methyl groups to each primary amine ${ }^{21}$. Methylated DBL3x was still recognized in immunoblotting by monoclonal antibodies raised against the native DBL3x (data not shown), consistent with the gentle nature of lysine methylation.

ITC experiments also demonstrated the binding of CSA by DBL3x. When $30 \mu \mathrm{M}$ bovine tracheal CSA was titrated into a solution of $30 \mu \mathrm{M}$ DBL3x in the same $100 \mathrm{mM} \mathrm{Na-HEPES}$ buffer used in crystallization, we observed binding (Supplementary Fig. 3 online). We could not detect binding when methylated DBL3x was titrated with bovine tracheal CSA or when unmodified wild-type DBL3x was titrated with unsaturated CSA disaccharides produced by chondroitinase $\mathrm{ABC}$ digestion (data not shown).

\section{Lysine mutations proximal to the CSA reduce binding}

In flow cytometry, we observed decreased CSA binding of a mutant DBL3x in which Lys1507 was replaced by alanine (Fig. 3c, purple peak). The use of a double mutant, with alanine substituted for both Lys1507 and Lys1510, additionally reduced the binding to the cells (Fig. 3c, green peak). In ITC experiments, both mutant DBL3x molecules yielded results consistent with the flow cytometry data (Supplementary Fig. 3). The titration data revealed a less favorable apparent enthalpy of binding of the mutants compared to wild-type DBL3x. Because the binding enthalpy is a direct measure of the interactions between bound ligand and protein relative to those existing when they are free in the solvent, the ITC results are evidence for the diminished strength of interaction between CSA and the DBL3x mutant molecules.

\section{Electrostatic potential surface of DBL3x}

The calculated electrostatic potential surface of DBL3x shows a patch of positive potential on the surface of the molecule near to the CSA electron density. Four lysine residues and two arginine residues contribute to the cluster of charges making up the patch (Fig. 4). The binding of CSA oligosaccharides seems to be largely electrostatic in nature, which is supported by our observation of decreased CSA binding to DBL3x with the addition of $50 \mathrm{mM} \mathrm{NaCl}$ to the $100 \mathrm{mM}$ Na-HEPES in ITC experiments (data not shown). The CSA oligosaccharides used for the crystal structure consist of negatively charged repeating disaccharides (Methods and Discussion). We have located one of the sulfates in the electron density as described above, which implicates the sulfate groups in binding to DBL3x. The DBL3x surface also shows that there is a patch of negative electrostatic potential at the bottom of the 'valley' between subdomains 2 and 3 contributed by a cluster of aspartic and glutamic acid residues (Fig. 4). The presence of 
Figure 4 The electrostatic potential surface in the vicinity of the CSA binding site. (a) Ribbon diagram of DBL3x overlayed on a semitransparent electrostatic potential surface. Side chains of lysines and arginines responsible for the positively charged region are shown in red. Subdomain 1 (yellow), subdomain 2 (blue) and subdomain 3 (red) are shown. For clarity, Lys1327 is not shown. (b) Positively charged areas (blue) are located on the sides of the binding area for the CSA oligosaccharide, shown in same orientation as in a. Lysines and arginines
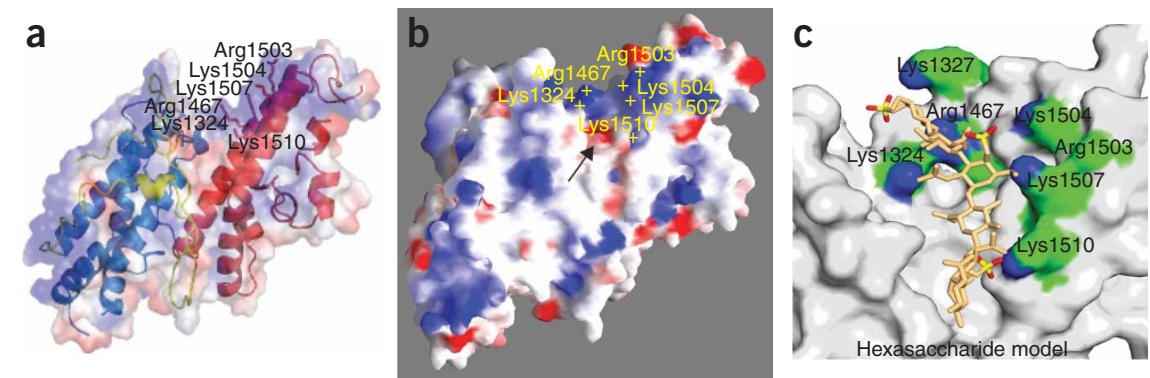

with side chains within $5 \AA$ of the carbohydrate density are labeled (+, yellow). Below the CSA binding site is a region of negatively charged residues (red at arrow), which could prevent CSA from occupying the deeper portions of the 'valley' between subdomains 2 and 3 . (c) View of a CSA hexasaccharide model 39 (PDB 1C4S) placed along the extra electron density seen in the crystals. The hexasaccharide shown here is approximately $30 \AA$ in length and similar to the length of the observed electron density. Lys 1327 is shown.

this negatively charged area would make the binding of negatively charged CSA energetically unfavorable. This would explain the location of the binding site on the top surface of the two helices of subdomain 3 and at the loop between helix $\mathrm{H} 1$ and $\mathrm{H} 2$ in subdomain 2. To clarify the extent of the binding site, we positioned a CSA hexasaccharide model as a rigid body along the location of the CSA electron density, superimposing a sulfate group from the CSA model on the sulfate identified experimentally in the electron density map (Fig. 4c).

\section{DISCUSSION}

We have described the structure of the CSA binding DBL3x domain of VAR2CSA, a parasite-encoded member of the PfEMP1 family of proteins expressed on the surface of infected erythrocytes and responsible for binding infected erythrocytes in the placenta. It contains three subdomains that are analogous to those described for the Pk $\alpha-\mathrm{DBL}$ domain $^{16}$ and for the two DBL domains found in EBA-175 (ref. 20), both non-PfEMP1 proteins involved in parasite invasion of erythrocytes. Notably, we have identified the region in DBL3x that binds CSA to be located in a valley between subdomains 2 and 3 .

Chondroitin sulfate chains are unbranched polysaccharides of variable length, sulfate content and sulfation pattern. They are composed of repeating disaccharides of alternating glucuronic acid and $\mathrm{N}$-acetylgalactosamine. Each $\mathrm{N}$-acetylgalactosamine may be nonsulfated or sulfated, most commonly at the 4-O or 6-O position. Human placental CS contains 4-O-sulfate on about $10 \%$ of $\mathrm{N}$-acetylgalactosamines and does not contain 6-O-sulfate ${ }^{22}$. Infected erythrocytes bind to chondroitin sulfate proteoglycans that have 4-O-sulfates, and the binding can be inhibited by soluble 4-O-sulfated chondroitin sulfate, but not by $6-O$-sulfated chondroitin sulfate ${ }^{14}$. In this work, we used bovine tracheal CSA (a copolymer of 53\% 4-O-sulfate, CSA, and 39\% 6-O-sulfate, CSC) from which the 6-O-sulfate groups were removed chemically and fractionated by ion-exchange chromatography to obtain partially sulfated CS fractions of varying 4-O-sulfate contents ${ }^{23}$. Several sizes of oligosaccharides obtained from a CS fraction containing 50\% 4-O-sulfated and 50\% nonsulfated disaccharide moieties could bind to the DBL3x domain in the crystal. Furthermore, a hexasaccharide fraction inhibited the binding of DBL3x to CSA expressed on the surface of $\mathrm{CHO}$ cells. Another X-ray structure of the DBL3x domain was recently reported $^{24}$. In that work, soaking crystals with ammonium sulfate or with the mixture of unsaturated CSA and CSC disaccharides obtained by bacterial chondroitinase $\mathrm{ABC}$ digestion of bovine trachea CSA showed a sulfate bound at the same location as the sulfate bound to DBL3x in the present study. Electron density for an unsaturated disaccharide was not observed ${ }^{24}$, which is consistent with our finding that unsaturated CSA disaccharides did not compete with CSA for binding to DBL3x.

The heterogeneity in size and charge density of glycosaminoglycans hinders the determination of the structural basis of their interaction with proteins. The lack of a well-defined and continuous electron density for the different CSA oligosaccharides studied here could be due to the presence of multiple modes of binding, partial disordering, conformational fluctuations or chemical heterogeneity. Charged oligosaccharides that interact with proteins may be held in place predominantly by charge interactions without many other substantial interactions. In defining the binding of a heparin-like ligand to a thrombospondin domain, the density of the bound heparin-like molecule was also not well defined ${ }^{25,26}$, but sulfates could be positioned in the largest electron density, as in this work. We speculate that the flexible oligosaccharides bound to DBL3x may be mobile, with mainly charged groups from both protein and ligand involved in binding.

When two of the lysines (Lys1507 and Lys1510) in proximity to the CSA density were mutated to alanine, flow cytometry and ITC revealed substantially decreased binding of the mutant DBL3x proteins to soluble CSA. Furthermore, a 67-residue peptide from a DBL3 $\gamma$ domain, which corresponds to the $\mathrm{H} 5$ and H6 helices of the DBL3x subdomain 3 , has been shown to bind CSA as efficiently as the entire DBL3 $\gamma$ domain $^{27}$ (Supplementary Fig. 4 online). Taken together, these data indicate that the extra electron density observed in this work indeed comes from CSA bound to DBL3x. This region is highly conserved in all VAR2CSA DBL3x domains ${ }^{28}$.

This mode of binding, which involves only surface-exposed residues and does not seem to involve nonbonding (van der Waals) interactions, is consistent with the small differences between the native and complexed structures of DBL3x. When superimposing the two models based on the $\mathrm{C} \alpha$ positions of all helices, the r.m.s. deviation is only $0.9 \AA$ A . Overlaying the two models using only the $\mathrm{C} \alpha$ atoms of helices H5 and H6, the r.m.s. deviation is $0.4 \AA$ between these helices in each model. The low r.m.s. deviation implies that CSA binding to the charged surface of DBL3x does not alter the protein structure. When the models are aligned by $\mathrm{H} 5$ and $\mathrm{H} 6$, the r.m.s. deviation between the coordinates of both subdomain 2 regions is $0.8 \AA$. We do not see flexibility or movement of the subdomains relative to each other.

The results of our study provide a structure-based explanation for how the interaction between CSA and DBL3x depends on the overall charge distribution in the protein fold. The specificity of CSA binding may be mediated not just by clusters of basic residues but also by their particular location in the tertiary structure of the protein. The 
specificity of binding of CSA to DBL3x is probably due to the coordination of 4-O-sulfate groups and to a particular conformation of CSA that is favored by the distribution of charged residues on the protein.

As a target for vaccine development to protect pregnant women from malaria, it is important to note that the second helix of subdomain 3 of DBL3x contains highly conserved residues ${ }^{28,29}$. This conservation, especially for a receptor under immune selection, is highly unusual in P. falciparum. However, conservation has been suspected owing to the finding that women become immune to VAR2CSA after their first pregnancy ${ }^{15}$ and consequently are protected from pregnancy-associated malaria. Thus, a vaccine designed using DBL3x structural information may elicit an immune response that would effectively block the binding of infected erythrocytes in the placenta during pregnancy.

\section{METHODS}

Protein production. We cloned the DBL3x (residues 1220-1580, GenBank AAQ73926) from genomic DNA of the A4 strain (also called FCR3 or IT) of P. falciparum in the pLM1 expression plasmid ${ }^{30}$. The plasmid was transformed into BL21(DE3)-RIL cells that were then grown to an optical density at $600 \mathrm{~nm}$ $\left(\mathrm{OD}_{600}\right)$ of 0.8 and induced with $1 \mathrm{mM}$ IPTG to produce insoluble protein as inclusion bodies. Solubilized inclusion bodies were refolded at $4{ }^{\circ} \mathrm{C}$ in 1 liter of buffer containing $50 \mathrm{mM}$ Tris- $\mathrm{HCl}, \mathrm{pH}$ 7.6, $50 \mathrm{mM} \mathrm{NaCl}, 500 \mathrm{mM}$ arginine$\mathrm{HCl}, 2 \mathrm{mM}$ EDTA, $40 \mathrm{mM}$ sucrose, $2 \mathrm{mM}$ DTT and $5 \mathrm{mM}$ cystamine-HCl. After refolding for $12-15 \mathrm{~h}$, we added $11 \mathrm{~g}$ cystamine- $\mathrm{HCl}$ to react with free cysteines, and the refolded protein was dialyzed against $20 \mathrm{mM}$ Tris- $\mathrm{HCl}, \mathrm{pH} 7.5$. We purified refolded DBL3x using cation-exchange chromatography and size-exclusion chromatography. Purified DBL3x was dialyzed against $10 \mathrm{mM}$ Tris- $\mathrm{HCl}, \mathrm{pH} 7.5$, at $4{ }^{\circ} \mathrm{C}$ and concentrated to $10 \mathrm{mg} \mathrm{ml}^{-1}$ for crystallization.

We used standard techniques to add the six-histidine tag to the $\mathrm{C}$ terminus and to mutate DBL3x. The His-tagged and mutant proteins were expressed and refolded under the same conditions as the wild-type protein. The refolded protein solution was adjusted to the binding buffer conditions ( $20 \mathrm{mM}$ sodium phosphate, $0.5 \mathrm{M} \mathrm{NaCl}, 20 \mathrm{mM}$ imidazole- $\mathrm{HCl}, \mathrm{pH}$ 7.4). The protein was then bound to a nickel-nitrilotriacetic acid (Ni-NTA) Sepharose column and eluted with a gradient of $0.5 \mathrm{M}$ imidazole- $\mathrm{HCl}$ in the binding buffer. The protein was further purified by size-exclusion chromatography, then dialyzed against $10 \mathrm{mM}$ Tris- $\mathrm{HCl}, \mathrm{pH} 7.5$, at $4{ }^{\circ} \mathrm{C}$ and concentrated to $20 \mathrm{mg} \mathrm{ml}^{-1}$. DBL3x was exhaustively methylated as described ${ }^{31}$. MS of methylated DBL3x confirmed the addition of two methyl groups to each of the 50 lysines and to the $\mathrm{N}$ terminus.

Preparation of size-fractionated and partially desulfated oligosaccharides from bovine tracheal CSA. Bovine tracheal CSA, desalted on Dowex 50W-X8 $\left(\mathrm{H}^{+}\right)$resin, was neutralized with pyridine and lyophilized. The pyridinium salt of CSA thus obtained was 6-O-desulfated using $\mathrm{N}$, O-bis(trimethylsilyl)acetamide in pyridine and fractionated on DEAE-Sephacel columns as described previously ${ }^{14,23}$. The chondroitin 4-O-sulfate fraction containing 50\% 4-Osulfated and $50 \%$ nonsulfated disaccharide moieties was partially depolymerized with ovine testicular hyaluronidase and the oligosaccharides of varying sizes that formed were isolated by size-exclusion chromatography on Bio-Gel P-6 (ref. 14).

Crystallization of DBL3x. Crystals were grown using the hanging-drop method by mixing $1 \mu \mathrm{l}$ of protein and $1 \mu \mathrm{l}$ of precipitant and equilibrating against $500 \mu \mathrm{l}$ of precipitant. Crystals appeared with $20-25 \%$ (w/v) PEG3350 in $100 \mathrm{mM}$ Na-HEPES buffer at $\mathrm{pH} 7.5$ in $2-3$ weeks. When grown in the presence of 4-10 $\mathrm{g} \mathrm{ml}^{-1}$ of high-molecular-weight CSA (bovine tracheal CSA, Sigma-Aldrich C9819) crystals appeared more quickly, in 4-5 d. Native crystals and crystals used for soaking grew in the presence of this low concentration of high-molecular-weight CSA.

We performed cocrystallization experiments using DBL3x and CSA oligosaccharides ranging in size from 4 to 14 monosaccharides for various concentrations of oligosaccharides (1-10 mM). Experiments with oligosaccharides having four, six or eight monosaccharides yielded cocrystals under the same conditions as native crystals. Cocrystals were briefly cryoprotected with
Table 1 Data collection and refinement statistics

Native DBL3x

Complexed with CSA

Data collection

Space group

Cell dimensions

$a, b, c(\AA)$

Resolution $(\AA)$

$R_{\text {sym }}$

$I / \sigma I$

Completeness (\%)

Redundancy

$P 2{ }_{1}{ }_{1} 2_{1}$

$P 2{ }_{1}{ }_{1} 2_{1}$

\section{Refinement}

Resolution $(\AA)$

No. reflections

$R_{\text {work }} / R_{\text {free }}$

No. atoms

Protein

Ligand

Water

$B$-factors

Protein

Water

$40.67,84.20,86.49$

40-1.9 (2.01-1.90)*

$42.52,86.76,101.57$

20-2.8 (2.97-2.80)

$6.9(43.5)$

$12.5(2.85)$

$16.6(2.4)$

$99.1(94.8)$

$98.5(98.0)$

3.7 (3.6)

R.m.s. deviations

Bond lengths ( $(\AA)$

Bond angles $\left({ }^{\circ}\right)$

$9.2(7.6)$

20-2.8

9717

$0.30 / 0.35$

$0.22 / 0.25$

2244

$5\left(\mathrm{SO}_{4}\right)$

98

65.0

40.8
40.3

$-$

*Values in parentheses are for highest-resolution shell.

35\% (w/v) PEG3350 in $100 \mathrm{mM} \mathrm{Na-HEPES,} \mathrm{pH} \mathrm{7.5,} \mathrm{before} \mathrm{freezing} \mathrm{the} \mathrm{crystals}$ in liquid $\mathrm{N}_{2}$.

Crystals were soaked with various concentrations (1-25 mM) of oligosaccharides from 4 to 14 monosaccharides in length, with soaking times of a few seconds up to $24 \mathrm{~h}$. The oligosaccharides were dissolved in the cryoprotecting solution of $35 \%$ (w/v) PEG3350, $100 \mathrm{mM} \mathrm{Na-HEPES,} \mathrm{pH} 7.5$, and soaking was performed before freezing in liquid nitrogen. All native DBL3x and DBL3x-CSA complex crystals were grown in identical buffer conditions, except that in the cocrystallizations we added CSA oligosaccharides instead of highmolecular-weight CSA.

Structure determinations. We collected $50 \mathrm{X}$-ray data sets and processed them with HKL2000 ${ }^{32}$ and $\mathrm{XDS}^{33}$. Our attempts to prepare heavy-atom derivatives were unsuccessful. Molecular replacement techniques were then pursued using the EBA-175 (PDB 1ZRL, PDB 1ZRO) and Pk $\alpha$-DBL (PDB 2C6J) structures as search models. Residues of the protein models were all changed to serine, except for the alanine and glycine residues. Loop residues were removed, keeping only the residues of the largest helices and part of subdomain 1 . The modified Pk $\alpha$-DBL model was positioned in the DBL3x unit cell based on the X-ray data from one of our early data sets $\left(P 2_{1} 2_{1} 2_{1}\right.$, unit cell of $39.98,83.34$, $85.95,90,90,90)$ using the maximum-likelihood molecular replacement algorithms of the PHASER software package ${ }^{34}$. Although the PHASER $Z$-score, the log-likelihood gain and packing considerations indicated that the positioning of the model was correct, electron-density maps produced with calculated phases did not reveal new protein density beyond the polyserine model. Similar quality solutions were obtained in three other X-ray datasets: $P 2{ }_{1} 2_{1} 2_{1}$, unit cell of $41.2186 .97,91.50,90,90,90 ; P 2_{1} 2_{1} 2_{1}$, unit cell of $39.91,86.07,86.95,90,90$, 90; and $P 2_{1}$, unit cell of $40.93,85.56,97.59,90,94.4,90$. Using a mask made from the polyserine model, the rotation and translation operators relating the molecular replacement solutions of each data set, and calculated phases in each data set, we carried out multicrystal averaging to $3.0 \AA$ using the DMMULTI software ${ }^{35}$. This markedly improved the electron density, yielding densities for side chains and disulfides and allowing the backbone tracing of the five largest helices and the assignment of side chains in the $\mathrm{H} 5$ and $\mathrm{H} 6$ helices. The model was further improved by cycles of 
manual building and refinement ${ }^{36}$ and completed in the native data shown in Table 1. Structures of DBL3x-CSA complexes were solved with the native model in PHASER, refined in $\mathrm{CNS}^{36}$ and extensively rebuilt in the $\mathrm{O}$ graphics program ${ }^{37}$.

Flow cytometry. The binding of DBL3x to CSA expressed on the surface of CHO K1 cells (American Type Culture Collection) was monitored using flow cytometry $^{38}$. Cells were harvested by trypsinization, washed, and suspended in FACS buffer (PBS containing 0.5\% (w/v) BSA and $0.1 \%(\mathrm{w} / \mathrm{v})$ sodium azide). About one million cells $(100 \mu \mathrm{l})$ were incubated for $30 \mathrm{~min}$ with $15 \mu \mathrm{M}$ of DBL3x either in the presence $(1 \mathrm{mM})$ or absence of bovine tracheal CSA or shark cartilage CSC (Seikagaku Corp., 400670) at room temperature $\left(20-22{ }^{\circ} \mathrm{C}\right)$. The bovine tracheal CSA was not 6-O-desulfated for the flow cytometry or ITC experiments. After incubation, cells were washed three times in FACS buffer and treated with anti-DBL3x mAb for $30 \mathrm{~min}$ at $4{ }^{\circ} \mathrm{C}$. The cells were washed twice in PBS and anti-DBL3x antibodies were detected with Alexa 488-labeled goat anti-mouse IgG (100 $\mu \mathrm{l})$ (Invitrogen, A11029) diluted 1:250 in FACS buffer. The cells were then incubated for $30 \mathrm{~min}$, washed, resuspended in $200 \mu \mathrm{l}$ FACS buffer and analyzed by FACSort (Becton Dickinson). All the incubations and washing steps were performed at $4{ }^{\circ} \mathrm{C}$. Events $(75,000)$ were acquired using CELLQuest software (version 3.3; Becton Dickinson), and the data from the live-cell gate were analyzed by FlowJo software (version 6.4.1; Tree Star).

Isothermal titration calorimetry. Wild-type and mutant DBL3x with His-tags were dialyzed extensively against filtered $(0.22 \mu \mathrm{m}) 100 \mathrm{mM} \mathrm{Na}$-HEPES buffer, $\mathrm{pH} 7.5$, and were diluted to $30 \mu \mathrm{M}$ in the same buffer. Lyophilized bovine tracheal CSA (reported molecular weight of 50,000 Da) was weighed and dissolved at $30 \mu \mathrm{M}$ in the same Na-HEPES buffer. The $100 \mathrm{mM}$ Na-HEPES, $\mathrm{pH}$ 7.5, buffer was diluted from a $1 \mathrm{M}$ solution of Na-HEPES (Hampton Research, HR2-733) that had been titrated to $\mathrm{pH} 7.5$ by the addition of $0.5 \mathrm{M} \mathrm{HCl}$. The buffer therefore contained $100 \mathrm{mM} \mathrm{Na}^{+}, 100 \mathrm{mM} \mathrm{HEPES}^{-}$(sulfonic acid), $50 \mathrm{mM} \mathrm{Cl}^{-}$and about $50 \mathrm{mM} \mathrm{HEPES}^{+}$(titratable amino group). All ITC experiments, crystallizations and crystal soakings used the identical $100 \mathrm{mM}$ Na-HEPES buffer.

We carried out calorimetric titrations using these solutions in a MicroCal VP-ITC microcalorimeter. The sample cell of the calorimeter was filled completely with the protein solution to a volume of $1.8 \mathrm{ml}$, and the system was allowed to equilibrate at $30{ }^{\circ} \mathrm{C}$. Aliquots $(10 \mu \mathrm{l})$ of the ligand solution were injected into the protein solution at 5-min intervals. Raw ITC data were integrated using the MicroCal Origin software, and background heats from titrations of CSA into buffer were subtracted to give corrected heats. Because of the heterogeneity of the CSA ligand, the parameters $n, K$ and $\Delta H$ could not be reliably derived from the data.

Accession codes. Protein Data Bank. Atomic coordinates and structure factors for the crystal structures of DBL3x alone and DBL3x complexed with CSA have been deposited under accesion codes $3 \mathrm{CML}$ and $3 \mathrm{CPZ}$, respectively.

Note: Supplementary information is available on the Nature Structural \& Molecular Biology website.

\section{ACKNOWLEDGMENTS}

We thank A. Diouf and C.A. Long (Laboratory of Malaria and Vector Research, National Institute of Allergy and Infectious Diseases) for screening monoclonal antibodies, J.F. Andersen for helping with ITC, S. Madala for giving assistance with flow cytometry, H.-P. Su for advising on molecular biology, J.M. Moore for helping with initial protein purification and J.D. Smith (Seattle Biomedical Research Institute) for giving us the A4 genomic DNA. X-ray data were collected at the SBC-CAT and SER-CAT beamlines at the Advanced Photon Source supported by the US Department of Energy, Office of Science, Office of Basic Energy Sciences, under contract W-31-109-Eng-38. This work was supported by the Intramural Research Program of the US National Institutes of Health, National Institute of Allergy and Infectious Diseases and by grant AI45086 for work performed in the laboratory of D.C. Gowda.

\section{AUTHOR CONTRIBUTIONS}

K.S. and L.H.M. initiated the project; K.S. and P.N. expressed the proteins and produced crystals; K.S., P.N. and A.G.G. collected X-ray data; K.S., A.G.G. and D.N.G. processed the X-ray data and solved the DBL3x structure; D.C.G. prepared desulfated, digested and size-fractionated CSA; K.S. and A.G.G. built models and refined structures for more than $20 \mathrm{X}$-ray data sets from crystals with carbohydrate; K.S., A.G.G., L.H.M. and D.N.G. wrote the manuscript. All authors edited the manuscript.

Published online at http://www.nature.com/nsmb/

Reprints and permissions information is available online at http://npg.nature.com/ reprintsandpermissions/

1. Gamain, B., Smith, J.D., Viebig, N.K., Gysin, J. \& Scherf, A. Pregnancy-associated malaria: parasite binding, natural immunity and vaccine development. Int. J. Parasitol. 37, 273-283 (2007).

2. Brabin, B.J. et al. The sick placenta-the role of malaria. Placenta 25, 359-378 (2004).

3. Hviid, L. \& Salanti, A. VAR2CSA and protective immunity against pregnancyassociated Plasmodium falciparum malaria. Parasitology 134, 1871-1876 (2007).

4. Beeson, J.G. \& Duffy, P.E. The immunology and pathogenesis of malaria during pregnancy. Curr. Top. Microbiol. Immunol. 297, 187-227 (2005).

5. Fried, M. \& Duffy, P.E. Adherence of Plasmodium falciparum to chondroitin sulfate A in the human placenta. Science 272, 1502-1504 (1996).

6. Rogerson, S.J., Chaiyaroj, S.C., Ng, K., Reeder, J.C. \& Brown, G.V. Chondroitin sulfate $A$ is a cell surface receptor for Plasmodium falciparum-infected erythrocytes. J. Exp. Med. 182, 15-20 (1995).

7. Fried, M., Domingo, G.J., Gowda, C.D., Mutabingwa, T.K. \& Duffy, P.E. Plasmodium falciparum: chondroitin sulfate $A$ is the major receptor for adhesion of parasitized erythrocytes in the placenta. Exp. Parasitol. 113, 36-42 (2006).

8. Salanti, A. et al. Selective upregulation of a single distinctly structured var gene in chondroitin sulphate A-adhering Plasmodium falciparum involved in pregnancyassociated malaria. Mol. Microbiol. 49, 179-191 (2003).

9. Fried, M. et al. The distinct proteome of placental malaria parasites. Mol. Biochem. Parasitol. 155, 57-65 (2007).

10. Viebig, N.K. et al. A single member of the Plasmodium falciparum var multigene family determines cytoadhesion to the placental receptor chondroitin sulphate A. EMBO Rep. 6, 775-781 (2005)

11. Duffy, M.F. et al. VAR2CSA is the principal ligand for chondroitin sulfate $A$ in two allogeneic isolates of Plasmodium falciparum. Mol. Biochem. Parasitol. 148, 117-124 (2006).

12. Gamain, B. et al. Identification of multiple chondroitin sulfate A (CSA)-binding domains in the var2CSA gene transcribed in CSA-binding parasites. J. Infect. Dis. 191, 1010-1013 (2005).

13. Avril, M. et al. Characterization of anti-var2CSA-PfEMP1 cytoadhesion inhibitory mouse monoclonal antibodies. Microbes Infect. 8, 2863-2871 (2006).

14. Alkhalil, A., Achur, R.N., Valiyaveettil, M., Ockenhouse, C.F. \& Gowda, D.C. Structural requirements for the adherence of Plasmodium falciparum-infected erythrocytes to chondroitin sulfate proteoglycans of human placenta. J. Biol. Chem. 275, 40357-40364 (2000)

15. Fried, M., Nosten, F., Brockman, A., Brabin, B.J. \& Duffy, P.E. Maternal antibodies block malaria. Nature 395, 851-852 (1998).

16. Singh, S.K., Hora, R., Belrhali, H., Chitnis, C.E. \& Sharma, A. Structural basis for Duffy recognition by the malaria parasite Duffy-binding-like domain. Nature 439, 741-744 (2006).

17. Smith, J.D. et al. Switches in expression of Plasmodium falciparum var genes correlate with changes in antigenic and cytoadherent phenotypes of infected erythrocytes. Cell 82, 101-110 (1995).

18. Su, X.Z. et al. The large diverse gene family var encodes proteins involved in cytoadherence and antigenic variation of Plasmodium falciparum-infected erythrocytes. Cell 82, 89-100 (1995).

19. Baruch, D.I. et al. Cloning the P. falciparum gene encoding PfEMP1, a malarial variant antigen and adherence receptor on the surface of parasitized human erythrocytes. Cell 82, 77-87 (1995).

20. Tolia, N.H., Enemark, E.J., Sim, B.K. \& Joshua-Tor, L. Structural basis for the EBA175 erythrocyte invasion pathway of the malaria parasite Plasmodium falciparum. Cell 122, 183-193 (2005).

21. Rayment, I. Reductive alkylation of lysine residues to alter crystallization properties of proteins. Methods Enzymol. 276, 171-179 (1997).

22. Achur, R.N., Valiyaveettil, M., Alkhalil, A., Ockenhouse, C.F. \& Gowda, D.C. Characterization of proteoglycans of human placenta and identification of unique chondroitin sulfate proteoglycans of the intervillous spaces that mediate the adherence of Plasmodium falciparum-infected erythrocytes to the placenta. J. Biol. Chem. 275 , 40344-40356 (2000).

23. Muthusamy, A. et al. Structural characterization of the bovine tracheal chondroitin sulfate chains and binding of Plasmodium falciparum-infected erythrocytes. Glycobiology 14, 635-645 (2004).

24. Higgins, M.K. The structure of a chondroitin sulphate-binding domain important in placental malaria. J. Biol. Chem. published online, doi:10.1074/jbc.C8000086200 (11 June 2008).

25. Tan, K. et al. Heparin-induced cis- and trans-dimerization modes of the thrombospondin-1 N-terminal domain. J. Biol. Chem. 283, 3932-3941 (2008).

26. Tan, K. et al. The structures of the thrombospondin-1 N-terminal domain and its complex with a synthetic pentameric heparin. Structure 14, 33-42 (2006). 
ARTICLES

27. Gamain, B. et al. Identification of a 67-amino-acid region of the Plasmodium falciparum variant surface antigen that binds chondroitin sulphate $A$ and elicits antibodies reactive with the surface of placental isolates. Mol. Microbiol. 53, 445-455 (2004).

28. Dahlback, M. et al. Epitope mapping and topographic analysis of VAR2CSA DBL3X involved in P. falciparum placental sequestration. PLoS Pathog. 2, e124 (2006).

29. Bockhorst, J. et al. Structural polymorphism and diversifying selection on the pregnancy malaria vaccine candidate VAR2CSA. Mol. Biochem. Parasitol. 155, 103-112 (2007)

30. Sodeoka, M., Larson, C.J., Chen, L., LeClair, K.P. \& Verdine, G.L. A multifunctional plasmid for protein expression by ECPCR: overproduction of the p50 subunit of NF-kB. Bioorg. Med. Chem. Lett. 3, 1089-1094 (1993).

31. Saxena, A.K., Saul, A. \& Garboczi, D.N. Crystallization and preliminary X-ray analysis of the Plasmodium vivax sexual stage $25 \mathrm{kDa}$ protein Pvs25, a transmission-blocking vaccine candidate for malaria. Acta Crystallogr. D Biol. Crystallogr. 60, 706-708 (2004).
32. Otwinowski, Z. \& Minor, W. Processing of X-ray diffraction data collected in oscillation mode. Methods Enzymol. 276, 307-326 (1997).

33. Kabsch, W. Automatic processing of rotation diffraction data from crystals of initially unknown symmetry and cell constants. J. Appl. Crystallogr. 26, 795-800 (1993).

34. McCoy, A.J. et al. Phaser crystallographic software. J. Appl. Cryst. 40, 658-674 (2007).

35. Collaborative Computational Project Number 4. The CCP4 suite: programs for protein crystallography. Acta Crystallogr. D Biol. Crystallogr. 50, 760-763 (1994).

36. Brunger, A.T. Version 1.2 of the Crystallography and NMR system. Nat. Protocols 2 2728-2733 (2007).

37. Jones, T.A. Interactive electron-density map interpretation: from INTER to O. Acta Crystallogr. D Biol. Crystallogr. 60, 2115-2125 (2004).

38. Gamain, B., Miller, L.H. \& Baruch, D.I. The surface variant antigens of Plasmodium falciparum contain cross-reactive epitopes. Proc. Natl. Acad. Sci. USA 98, 2664-2669 (2001).

39. Winter, W.T., Arnott, S., Isaac, D.H. \& Atkins, E.D. Chondroitin 4-sulfate: the structure of a sulfated glycosaminoglycan. J. Mol. Biol. 125, 1-19 (1978). 